\title{
VENTRICULAR PARASYSTOLE WITH SLOW MANIFEST ECTOPIC DISCHARGE
}

\author{
BY \\ L. SCHAMROTH \\ From the Baragwanath Hospital and University of the Witwatersrand, Johannesburg, South Africa \\ Received February 8, 1962
}

It is a fundamental law of cardiac physiology that the dominant or fastest pacemaker determines the heart rate. All other subsidiary or slower pacemakers are prematurely discharged by the impulses from the dominant pacemaker.

In parasystole, an ectopic pacemaker is in some way protected from the impulses of the dominant (usually the sinus) pacemaker. The ectopic pacemaker is therefore undisturbed by the sinus impulses and discharges regularly at its own inherent rate. Its discharge will only become manifest when it occurs outside the refractory period of the ventricles resulting from their stimulation by the dominant pacemaker. Parasystole is thus a dual rhythm where two pacemakers concurrently and independently govern the heart. The arrhythmia has the following characteristics.

(1) Presence of Ectopic Beats with Interectopic Intervals Mathematically Related to Each Other. An interectopic interval is defined as the interval between two ectopic beats containing intervening sinus beats: the ectopic cycle length is the interval between two ectopic beats without intervening sinus beats (Scherf and Schott, 1953). In parasystole some of the ectopic discharges are not manifest since they occur when the ventricles are refractory following stimulation by the sinus pacemaker. However, the ectopic pacemaker discharges regularly whether its impulses are manifest or not and thus the longer interectopic intervals are multiples of the shortest interectopic interval or of the ectopic cycle length. The shortest measurable interval between ectopic beats represents the manifest ectopic discharge rate.

(2) Presence of Varying Coupling Intervals. The coupling interval is the interval between the : ectopic beat and the preceding sinus beat. With ventricular extrasystoles the ectopic beat is in some way forced or precipitated by the preceding sinus beat: the ectopic beat will thus bear a constant relation to the preceding sinus beat, i.e. the coupling intervals are constant. In parasystole the ectopic pacemaker is autonomous and beats independently of the sinus pacemaker; consequently the relation between the ectopic beats and the sinus beats will vary, i.e. the coupling interval usually differs with each ectopic beat.

(3) Presence of Fusion Beats (also known as summation or combination beats). As the two pacemakers in parasystole discharge at their own inherent rates, occasional synchronous or near-syn, chronous discharge may occur. There will then be simultaneous invasion of the ventricular musculature by both impulses, each activating part of the ventricles. The resulting QRS complex has a configuration intermediate between the pure sinus and pure ventricular complexes. The resulting summation or combination complex is known as a fusion beat.

Occasionally a parasystolic impulse does not become manifest even if its timing is such that it occurs during the non-refractory phase of the ventricular cycle, viz. the ectopic focus discharges but does not invade the surrounding myocardium even though the myocardium is responsive. This

has been explained by postulating a conduction defect at the ectopic-ventricular (E-V) junction-a condition known as exit block (Kaufmann and Rothberger, 1919, 1920).

This report describes an unusual case of ventricular parasystole characterized by a very slow 
manifest ectopic discharge that fluctuated from day to day. The reason for this and the true discharge rate were revealed when, during a short period, temporary dissipation of a very high-grade exit block occurred.

\section{CASE RePORT}

The electrocardiograms are those of a 13-year-old African (negro) girl who had acute nephritis. There were no clinical complications and recovery was uneventful. Follow-up 10 months later showed the same cardiographic abnormalities but physical examination was normal in every respect. She was not given digitalis or any other cardiac drugs.

The cardiogram recorded on admission (Fig. 1, continuous strip of lead II, part of a 10-minute recording) shows a basic sinus rhythm with cycle lengths ranging from 66 to $89^{*}$. The rhythm is interspersed with ectopic beats of uniform shape. The coupling intervals vary from 52 to 80 . The spacing of the ectopic

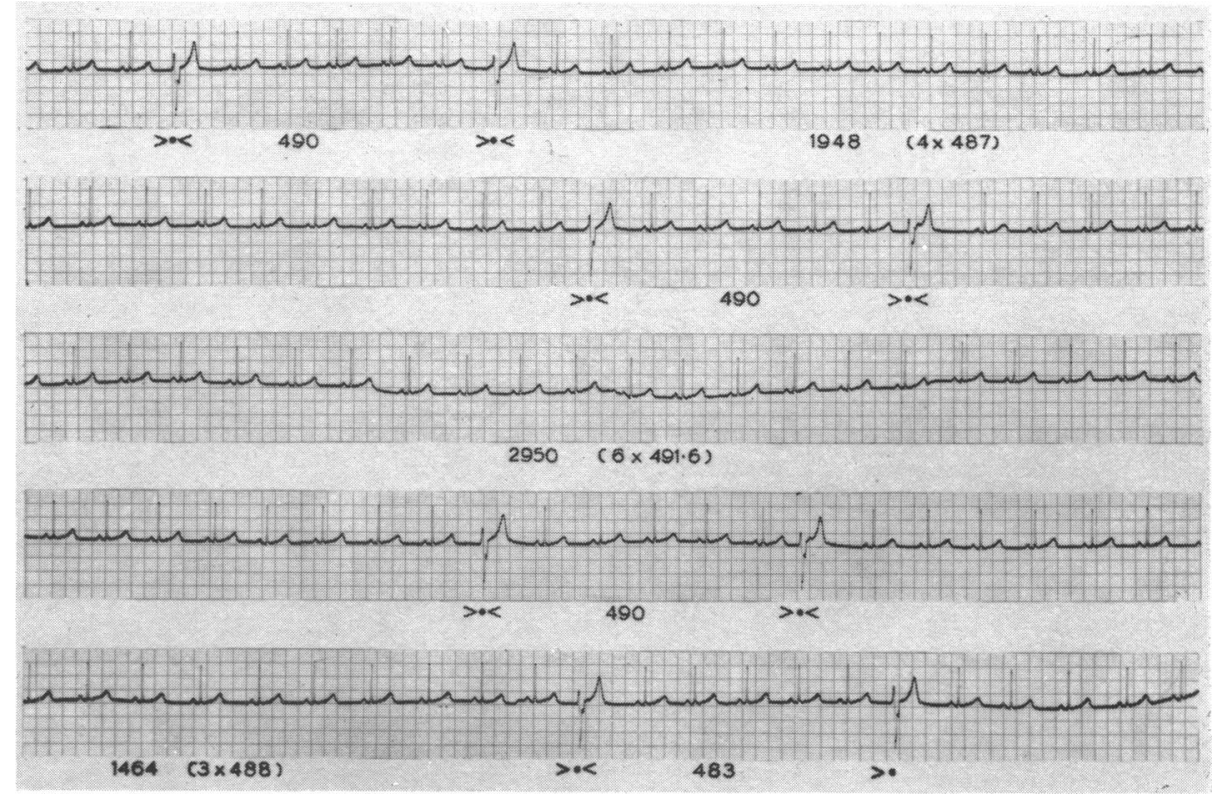

FIG. 1.-Electrocardiogram (continuous strip, standard lead II) showing parasystole with average interectopic interval of 488 . Time intervals are in hundredths of a second. Full description in text.

beats results in both short and long interectopic intervals. The shorter interectopic intervals in the whole recording ranged from 482 to 501 with close grouping around the mean of 488 . The long interectopic intervals were multiples or near-multiples of 488 . Occasional fusion beats (not shown in Fig. 1) were noted.

The presence of varying coupling intervals, interectopic intervals that are multiples of a common denominator, and fusion beats indicates a parasystolic rhythm.

Row I of Fig. 2 is a diagrammatic representation of the manifest arrhythmia. The sinus beats do not penetrate into the ectopic focus-EF in figure. The ectopic focus is thus protected and discharges manifestly at positions 1 and 2. The second discharge is synchronous with the sinus impulse and results in a fusion beat. A cardiogram recorded the next day showed the same arrhythmia.

A cardiogram recorded on the third day showed the same basic features as those described in Fig. 1 but in this recording the interectopic intervals were multiples or near multiples of 555-range 540 to 580 . Fig. 3, a continuous strip of lead V1, is a section of this 10-minute recording. Longer sections of the continuous recordings were submitted showing the wide range of fluctuation of the interectopic intervals; these were partially cut for editorial purposes.

* All time intervals are noted in hundredths of a second, i.e. 66 represents $0.66 \mathrm{sec}$. 

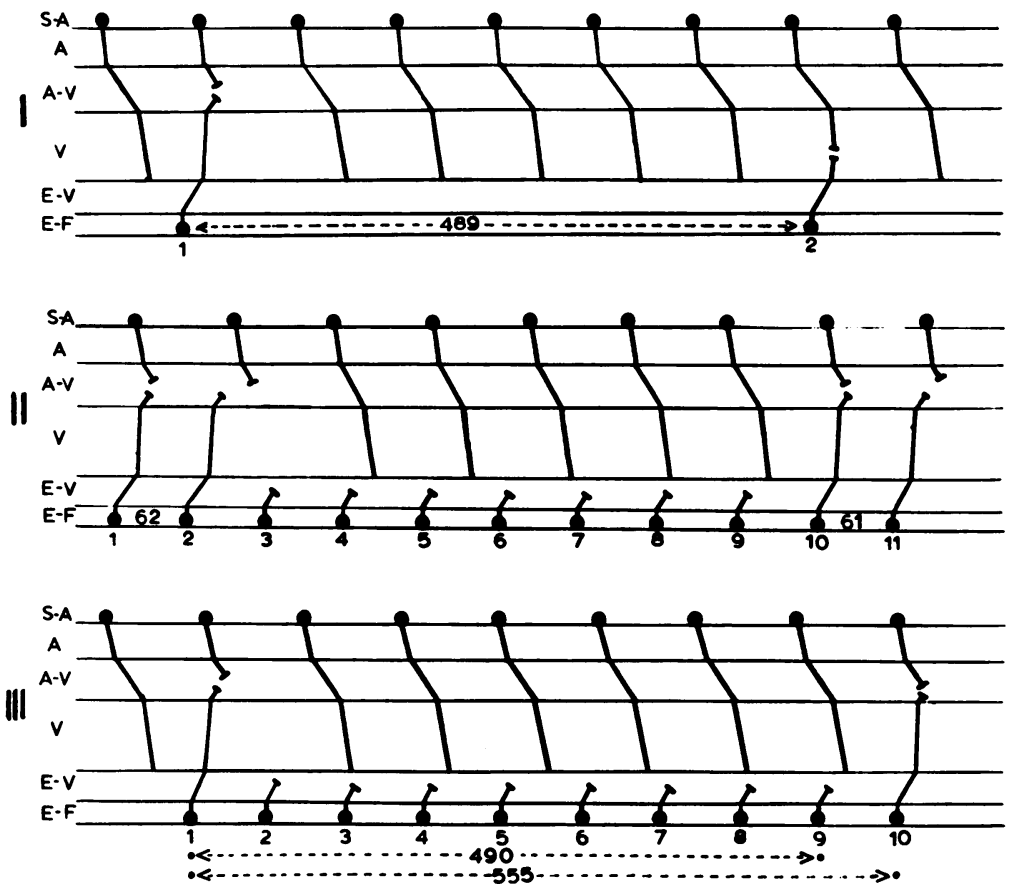

FIG. 2.-Diagrammatic representation of the various mechanisms occurring in this arrhythmia. Full description in text. S-A, sinu-atrial node; A, atrial level; A-V, atrio-nodal level; V, ventricular level; E-V, ectopic-ventricular junction; E-F, ectopic focus. Time intervals are in hundredths of a second.

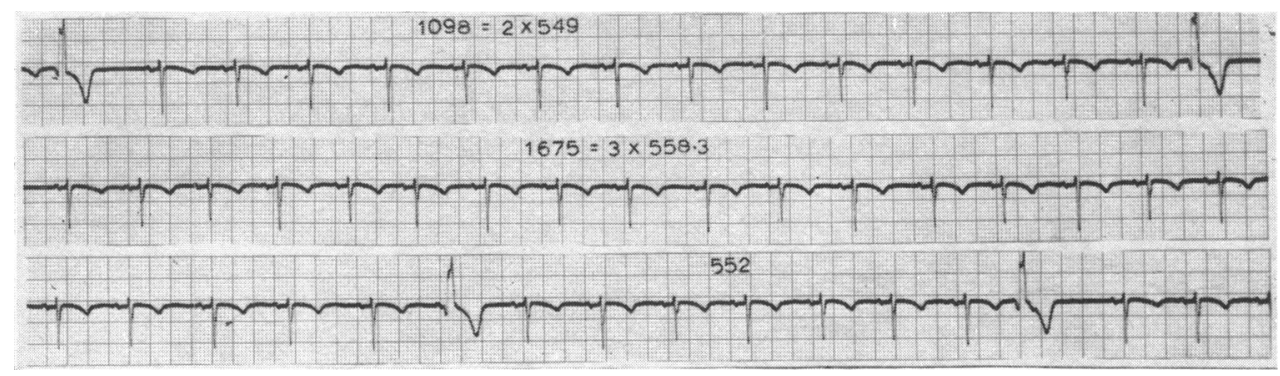

FIG. 3.-Electrocardiogram (continuous strip, lead V1) showing parasystole with average interectopic interval of 555. Time intervals are in hundredths of a second. Full description in text.

\section{Discussion}

Parasystole is usually associated with organic heart disease. In this case the arrhythmia was first noted during an attack of acute nephritis. However, the presence of the arrhythmia 10 months after the attack of nephritis, when the subject was normal in every other respect, suggests that the association was fortuitous.

Although the discharge rate of a parasystolic pacemaker is not always constant it varies much less than that of sinus rhythm; the fluctuation is usually within narrow limits and does not often exceed ten-hundredths of a second. The present case is remarkable in that, although the interectopic intervals were all multiples of a common denominator, the fluctuations in the same recording 
were relatively wide. In addition, the parasystolic discharge also fluctuated from day to day. Thus on some days it was in multiples of 488 with a range of from 482 to 501, and on other days it was in multiples of 555 with a range of from 540 to 580 .

Another remarkable feature of this case was the extremely slow manifest ectopic discharge rate which ranged from 10 to 12 a minute.

These phenomena became explicable on the sixth day in hospital when, during one short recording, additional cardiographic features appeared (Fig. 4). This tracing (continuous strip of lead V1, part of a two-minute recording) again shows the basic sinus rhythm interspersed with ectopic beats having an average interectopic interval 488 (top and bottom rows). However, the ectopic focus occasionally discharges in quick succession so that there are no intervening sinus beats (beats 1 and 2, 3 and 4, 6 and 7, and 8 and 9) thus revealing the ectopic cycle length. This was observed on five occasions and the resulting ectopic cycle lengths measured 62, 61, 62, 60, and 64 .

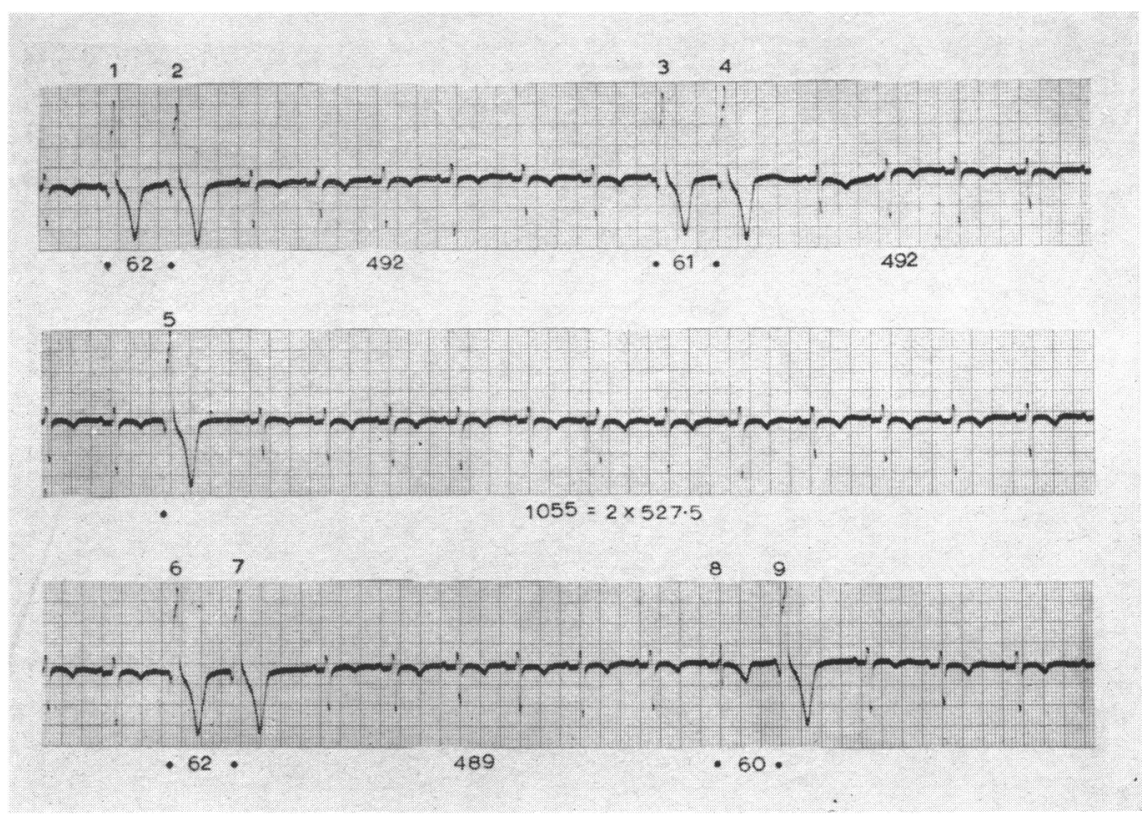

FIG. 4.-Electrocardiogram (continuous strip, lead V1) showing parasystole with average interectopic interval of 488 . Temporary dissipation of an exit block reveals the ectopic cycle length of 60 to 62 . Time intervals are given in hundredths of a second.

Analysis of the interectopic intervals of 488 and 555 shows that they are exact multiples or nearmultiples of 60 to 64 (usually 61 or 62 ). Thus $488=8 \times 61$, and $555=9 \times 61 \cdot 7$. The slow manifest ectopic rate is thus explicable on the basis of a relatively fast ectopic rhythm, discharging at a rate of 100 a minute but associated with a high grade exit block. When the manifest discharge rate is 488 , the exit block is $8: 1$; and when the manifest discharge rate is 555 , the exit block is $9: 1$. Momentary dissipation of the exit block (Fig. 4) results in $1: 1$ conduction thereby revealing the ectopic cycle length of 60.

The ectopic cycle length varied within very narrow limits: on the five occasions that it was measurable it ranged from 60 to 64 . It can readily be appreciated, however, that when this minor variation is multiplied by eight or nine (the degree of exit block) the range of fluctuation will be increased eight-or ninefold. Thus with an 8:1 exit block the fluctuation may range from $480(8 \times 60)$ to 512 $(8 \times 64)$; and with a $9: 1$ exit block the fluctuation may range from $540(9 \times 60)$ to $576(9 \times 64)$. It is 
thus apparent that, in the presence of but minor fluctuations in the ectopic cycle length, the higher the degree of exit block the wider the range of fluctuation in the manifest ectopic discharge.

These events are diagrammatically illustrated in row II of Fig. 2 which is representative of the top strip of Fig. 4. The ectopic focus, EF, discharges with a cycle length of 60 to 64 . A high-grade exit block exists as shown by the inability of impulses 3 to 9 to penetrate the ectopic-ventricular junction, EV. This exit block is occasionally dissipated allowing two successive ectopic discharges (impulses 1 and 2, and 10 and 11) to penetrate the ectopic-ventricular junction and activate the surrounding myocardium.

Row III of Fig. 2 is a diagrammatic illustration of the rhythm recorded in Fig. 3. The ectopic focus discharges with a cycle length of 60 . A high grade $(9: 1)$ exit block is present at the ectopic ventricular junction so that only impulses 1 and 10 penetrate the junction and become manifest; this results in an interectopic interval of 555. With an 8:1 exit block (cf. cardiogram in Fig. 1), impulses 1 and 9 will be manifest and the interectopic interval will be 488 .

The ectopic discharge of beat 8 in Fig. 4 results in a fusion beat. If this ectopic discharge had occurred but one- or two-hundredths of a second later it would not have been evident, as it would have discharged in the refractory period of the near-synchronous sinus beat. The manifest interectopic interval of 488 would thus have changed to a manifest interectopic interval of 555 . This illustrates how readily, in this recording, a manifest interectopic interval of 488 could become one of 555.

The long interectopic interval between impulses 5 and 6 in Fig. 4 is unusual. It measures 1055 which, when divided by 2 , results in 2 interectopic intervals of $527 \cdot 5$; this figure exceeds the possible range of the interectopic interval of 488 (range 480 to 512) and is well below the possible range of the interectopic interval of 555 (range 540 to 576). Further analysis, however, reveals that this interectopic interval could be composed of one interectopic interval in the 480 to 512 range, and another in the 540 to 576 range; for example, the interectopic interval could be a combination of 490 and 565 . This illustrates how an 8:1 exit block may fluctuate to a 9:1 exit block within one long interectopic interval.

Follow-up 10 months later showed the same basic arrhythmia but on this occasion the interectopic intervals were multiples or near-multiples of 429 -range 410 to 442 (Fig. 5). This indicates

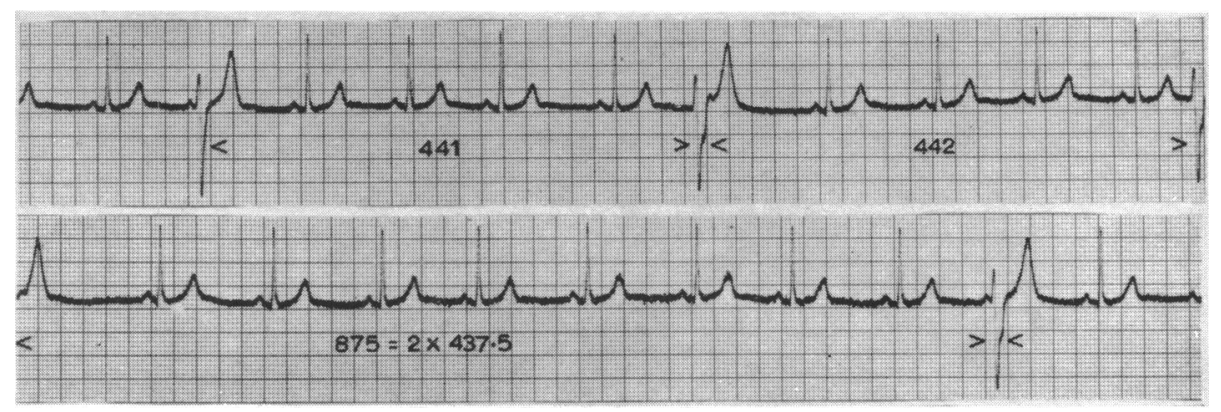

FIG. 5.-Electrocardiogram (continuous strip, standard lead II) showing parasystole; part of recording having average interectopic interval of 429 . Time intervals are in hundredths of a second. Full description in text.

the presence of a 7:1 exit block and suggests that the ectopic cycle length probably ranged from 59 to 64 ; thus $7 \times 59=413$, and $7 \times 64=448$. This could be diagrammatically represented in row III of Fig. 2 if impulses 1 and 8 were manifest. An hour later the rhythm reverted to a 9:1 exit block.

The concept of exit block has often to be invoked in the analysis of parasystolic rhythms. Its presence is inferred in cases with slow manifest rate where the calculated ectopic rate is faster than the sinus rate, otherwise an ectopic tachycardia would result. If no exit block were operative in this 
case, the true ectopic rhythm would become manifest at a rate of 100 a minute; and as this is faster than the sinus rate of 74 a minute, an ectopic tachycardia would result. Similarly, if the calculated ectopic cycle length is shorter than the compensatory pause following an ectopic beat, a continuous ectopic tachycardia would ensue unless an exit block were present. For example, in Fig. 4 the ectopic focus occasionally discharges manifestly for two beats in succession; the compensatory pause following the second discharge is much longer than the ectopic cycle length, and if exit block were not present the faster ectopic discharge would become the dominant ventricular pacemaker, resulting in an ectopic tachycardia. A report by Schott (1949) also illustrates this.

Exit block was also present in cases reported by Feher (1928), Faltitschek and Scherf (1932; their Case 6), Rosenblüth and Winterberg (1929), Scherf and Schott (1953; their Fig. 108 and 109), Schamroth and Marriott (1961; their Case 2), and Scherf and Bornemann (1961; their Cases 3, 4, 7, and 8).

The exit block is usually $2: 1$ but may occasionally be $3: 1$ or even $4: 1$. The degree of exit block may also fluctuate in the same recording. Thus in three of the cases described by Scherf and Bornemann (1961) the manifest ectopic discharge suddenly doubled suggesting momentary dissipation of a 2:1 exit block; the fourth case showed evidence of $2: 1,3: 1$, and possibly $4: 1$ exit block in the same recording. (In an addendum, they list three more cases where there was sudden doubling of the ectopic discharge rate.)

Dissipation of an exit block may only be momentary. In the present case it was only evident on five occasions during one short recording; in the case described by Schamroth and Marriott (1961) it was only evident twice.

Parasystole with exit block is also present when, during paroxysms of paroxysmal ventricular tachycardia, the pause between paroxysms is a multiple of the ectopic cycle length (Vedoya and Rodriguez Battini, 1939; Katz and Pick, 1956; their Fig. 194, 196, and 214).

Exit block makes a fast ectopic rhythm appear slow, and Scherf and Bornemann (1961) inferred that an exit block probably exists in many cases of parasystole. They also postulate that the rapid discharge of the ectopic centre makes it refractory to other impulses and that this alone is sufficient for its protection.

The present case supports these postulates; the parasystole is remarkable and unusual for the very high grade of exit block and the consequent very slow discharge rate. It emphasizes how an exit block may mask a fast ectopic rate.

While a mathematical relation between interectopic intervals is essential to the diagnosis of parasystole, the presence of a high grade exit block may exaggerate minor fluctuations in the true ectopic discharge and disturb the manifest precision of this relation. Furthermore, fluctuations in the degree of exit block within a single tracing and particularly during a long interectopic interval may also result in interectopic intervals that are apparently unrelated. These factors may mask the regularity of the true ectopic discharge.

\section{SUMMARY}

A case of ventricular parasystole with very slow manifest ectopic discharge is described. The interectopic intervals in a single recording fluctuated within relatively wide limits. The manifest ectopic discharge also fluctuated from day to day. These phenomena could be explained on the basis of a relatively fast ectopic discharge associated with a high grade, 7:1, 8:1, or 9:1 exit block.

I would like to thank Dr. I. Frack, Superintendent, Baragwanath Hospital, for permission to publish this case, and the Photographic Unit, Department of Medicine, University of the Witwatersrand, for the photographic reproductions.

\section{REFERENCES}

Faltitschek, F., and Scherf, D. (1932). Wien. Arch. inn. Med., 23, 269.

Feher, S. (1928). Wien. Arch. inn. Med., 15, 29.

Katz, L. N., and Pick, A. (1956). Clinical Electrocardiography, Part I. The Arrhythmias. Lea and Febiger, Philadelphia. 
Kaufmann, R., and Rothberger, C. J. (1919). Z. ges. exp. Med., 7, 199.

R, (1920). Z. ges. exp. Med., 11, 40.

Rosenblüth, E., and Winterberg, H. (1929). Wien. Arch. inn. Med., 16, 333.

Schamroth, L., and Marriott, H. J. L. (1961). Amer. J. Cardiol., 7, 799.

Scherf, D., and Bornemann, C. (1961). Amer. Heart J., 62, 320.

, and Schott, A. (1953). Extrasystoles and Allied Arrhythmias. Heinemann, London.

Schott, A. (1949). Trans. med. Soc. Lond., 65, 253.

Vedoya, R., and Rodriguez Battini, A. (1939). Rev. argent. Cardiol., 6, 313. 